\title{
AVANCES EN MANIPULACIÓN ROBÓTICA SUBMARINA MEDIANTE SEGMENTACIÓN Y SEGUIMIENTO VISUAL
}

\author{
David Fornas $^{\dagger}$, J. Javier Fernández ${ }^{\dagger}$, Antonio Soriano ${ }^{\ddagger}$, Diego Centelles ${ }^{\dagger}$, Pedro J. Sanz ${ }^{\dagger}$ \\ \{dfornas, fernandj, centelld, sanzp\}@uji.es, antonio.soriano-asensi@uv.es \\ $\dagger$ Departamento de Ingeniería y Ciencia de los Computadores. Universitat Jaume I. \\ Av. Sos Baynat, s/n, 12071 Castellón de la Plana (España) \\ ‡ Departament d'Informàtica. Universitat de València. Av. de la Universitat, s/n. 46100 Burjassot (España)
}

\section{Resumen}

En este artículo se presenta un sistema robótico semiautomático que utiliza visión, reconstrucción $3 D$, segmentación, estimación de objetos, interfaces hombre-robot (HRI) y planificación del agarre para mejorar el estado del arte en manipulación submarina mediante funciones autónomas y capacidad de supervisión. En primer lugar se obtiene una reconstrucción en $3 D$ del objeto. A continuación, se utilizan métodos basados en RANSAC (RANdom SAmple Consesnsus) y estimación de supercuádricas (SQ) para obtener la geometría subyacente de los objetos a manipular para presentar una reconstrucción de la escena al usuario. Después, se lleva a cabo la especificación de la manipulación de forma autónoma. El usuario puede entonces validar o ajustar el resultado del sistema y proceder a ejecutar el agarre. El sistema ha sido probado satisfactoriamente en diferentes condiciones, tanto en simulación como en entornos reales.

Palabras clave: reconstrucción 3D, estimación de la posición, supercuádricas, manipulación, robótica.

\section{INTRODUCCIÓN}

El uso de robots en el entorno submarino está creciendo notablemente: desde la industria del gas o el petróleo a la arquelogía o las ciencias marinas. El tipo de operaciones realizadas en estos sectores han sido llevadas acabo tradicionalmente con ROVs (Remote Operated Vechicle). Sin embargo, la tendencia actual está en la utilización de sistemas robóticos cada vez más autónomos con el objetivo de reducir los costes de operación. De este modo, recientemente han aparecido los I-AUVs (Intervention Autonomous Underwater Vehicle), aunque su utilización de momento se limita principalmente a proyectos de investigación [1] ya que la manipulación submarina sigue suponiendo un auténtico desafío. Una aproximación a medio camino entre los ROVs y los I-AUVs son los denominados HROVs (Hybrid ROVs), que son esencialmente I-AUVs reconfigurables como ROVs cuando se teleoperan remotamente a través de un cable umbilical o mediante comunicaciones inalámbricas. Probablemente, la demostración más avanzada de este tipo de sistemas en la actualidad sea el Ocean One Robotic Avatar [2], satisfactoriamente testeado en una misión arqueológica submarina de recuperación de objetos. Sin embargo, los costes de operación de dicho sistema aún son muy elevados.

El presente trabajo se enmarca en el el contexto del proyecto nacional coordinado MERBOTS, en el que participan la Universitat Jaume I (UJI), la Universitat de Girona (UdG) y la Universitat de les Illes Balears (UIB). El objetivo de MERBOTS es progresar en el desarrollo de sistemas de intervención submarina seguros, económicos y sencillos de utilizar en el contexto de la arqueología submarina. Concretamente, este trabajo se centra en el subproyecto MERMANIP dentro de MERBOTS, liderado por la UJI y dedicado, entre otros objetivos, al desarrollo de utilidades para la asistencia al arqueólogo durante las intervenciones de manipulación robótica submarina.

La manipulación robótica es un campo ampliamente estudiado desde hace décadas pero sigue siendo un campo muy activo y de gran interés para los investigadores, puesto que aún quedan bastantes problemas por resolver y avances por realizar. La planificación del agarre en entornos complejos no estructurados es uno de estos temas en los que se necesita avanzar. En un entorno estructurado, una de las aproximaciones más comunes en la planificación del agarre es la utilización del paradigma basado en modelo, es decir, utilizar leyes físicas para modelar la forma del objeto, los contactos y las fuerzas [3]. Algunos investigadores se han centrado en el análisis de agarres (el estudio de las propiedades físicas de un determinado agarre) y la síntesis de agarres (el cálculo de los agarres que mejor se adaptan a ciertas características deseables). Desafortunadamente, estas dos aproximaciones no funcionan en la práctica en escenarios complejos y con incertidumbre (p.e. escenarios submarinos) porque se basan principalmente en la suposición de tener un escenario estático. Por ejemplo, para el caso particular de un escenario 
submarino en aguas poco profundas, esta suposición no se cumple al tener una escena que no es estática, donde la posición relativa entre los objetos y el robot puede variar dinámicamente debido a las corrientes y al oleaje, o las condiciones de iluminación no son constantes variando en función de la nubosidad y las partículas en suspensión, etc.

Para afrontar estos inconvenientes, la solución más utilizada es incrementar la autonomía del sistema robótico mediante la incorporación de información sensorial. De entre toda esta información sensorial, la reconstrucción $3 \mathrm{D}$ de la escena es una de las más importantes para la planificación y la síntesis de agarres. Los sensores RGB-D ampliamente empleados en escenarios terrestres no son viables en entornos submarinos debido a la fuerte atenuación que sufre la luz infrarroja por el efecto del agua. Una alternativa viable es la utilización de cámaras estéreo, aunque tienen el inconveniente de funcionar sólo en escenarios texturizados. Otra alternativa son los sistemas de reconstrucción mediante láser, que requieren de un escaneado activo del objeto. En [4] se comparan las reconstrucciones 3D obtenidas mediante un sistema de visión estéreo y un sistema de reconstrucción láser bajo diferentes condiciones de luminosidad. En este trabajo, se utiliza información 3D obtenida mediante una cámara estéreo (ver Figura 1).

A partir de la reconstrucción 3D de la escena proporcionada por dichos sensores, existen diversas metodologías para la segmentación y el seguimiento de los objetos. De entre las que suponen que existe un conocimiento previo de los objetos a coger a través de modelos 3D de los mismos, en [5] se utiliza un algoritmo de detección basado en características. También, la RTM Toolbox [6] es un sistema de adquisición de objetos a partir de vistas parciales 3D que puede ser utilizado posteriormente para la estimación de la posición y el seguimiento.

En este trabajo se muestran el uso de RANSAC [7] y estimación de supercuádricas (SQ) [8] para extraer la forma aproximada de los objetos con el objetivo de planificar su manipulación. Para ello, proponemos métodos que no consideran la disponibilidad a priori de un modelo 3D del objeto, sino que utilizan modelos matemáticos a partir del conocimiento de información cualitativa relativa a la forma del objeto (esfera, cilindro, caja, etc.). La método basada en RANSAC es particularmente adecuada en el entorno de la arqueología submarina, ya que muchos objetos como las ánforas, cañones, etc. tienen formas básicas predominantes que se asemejan a cilindros, esferas u otras formas primitivas. Por otro lado, el método basado en SQ también es capaz de obtener modelos adecuados de este tipo de formas.

La siguiente sección describe el sistema de visión utilizado para la obtención de la posición de los objetos. En la Sección 3, la interfaz de usuario y los detalles de la manipulación son explicados mientras que la configuración experimental y los resultados son mostrados en la Sección 4. Finalmente, algunas conclusiones y posibilidades de trabajo futuro son proporcionados en la Sección 5 .

\section{ESTIMACIÓN DE LA POSICIÓN DE LOS OBJETOS}

En este trabajo proponemos el uso de técnicas de visión para la segmentación de los objetos y el fondo marino. Posteriormente se calcula un modelo geométrico de la forma de cada objeto, con el fin de estimar el agarre óptimo del mismo. Después de que la nube de puntos sea obtenida, ésta es segmentada para eliminar el fondo y se calcula un modelo geométrico del objeto que aproxima su forma. Además, el sistema de visión dispone de un sistema de seguimiento del movimiento del objeto durante la ejecución del agarre. En este sistema se proponen diferentes métodos que el usuario del sistema puede seleccionar para obtener la posición de forma satisfactoria. Los diferentes métodos se engloban en dos categorías: segmentación utilizando RANSAC y obtención del modelo mediante SQ.

\subsection{ESTIMACIÓN MEDIANTE RANSAC}

El método propuesto en primer lugar se basa en la utilización de RANSAC ${ }^{1}$ para obtener el modelo del objeto en varios paso. Se utiliza en primer lugar para extraer el fondo mediante la segmentación de un plano. Con este método se puede eliminar el fondo de forma aproximada y acelerar en gran medida los cálculos siguientes. En segundo

\footnotetext{
${ }^{1}$ Disponible en PCL (Point Cloud Library), una librería de tratamiento de nubes de puntos http: //pointclouds.org/.
}

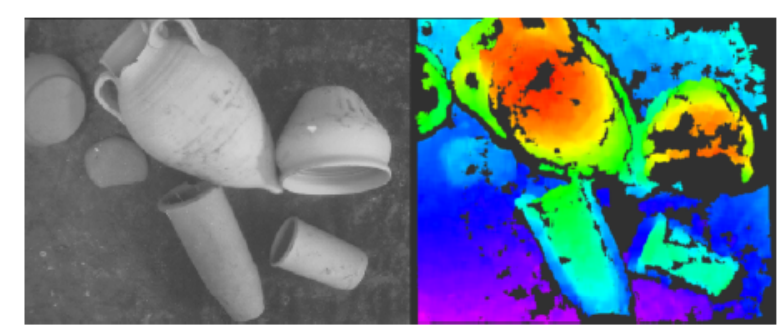

Figura 1: Escena con diferentes vasijas utilizadas en uno de los experimentos (izquierda). Mapa de profundidad de la escena obtenido con la cámara estéreo (derecha). 


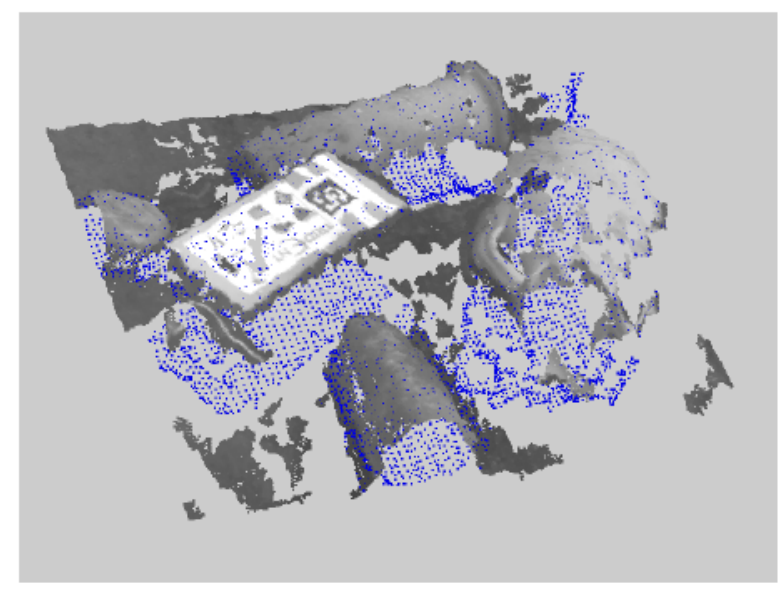

Figura 2: Sobre la nube de puntos de la escena se muestra en azul las partes de los objeto obtenidas mediante planos de simetría que ayudan a obtener las SQ.

lugar se utiliza para extraer el modelo del objeto, utilizando un cilindro, una esfera u otro plano para obtener las características de una caja. En este trabajo se amplia la metodología detallada en [9] con la segmentación de esferas y cajas. El uso de RANSAC permite encontrar las características de los objetos incluso en presencia de ruido. Se obtiene el radio y la altura del cilindro, el radio de la esfera y las dimensiones de la caja. Además, se obtiene la posición y orientación de todos los objetos (ver Figura 1).

\subsection{ESTIMACIÓN MEDIANTE SUPERCUÁDRICAS}

Alternativamente, se puede obtener el modelo del objeto utilizando modelos de SQ. La solución basada en SQ no requiere especificar la forma del objeto modelizado como ocurre en RANSAC. De esta forma, el uso de SQ mejora la autonomía de la planificación del agarre respecto a RANSAC.

Las supercuádricas son una familia de formas geométricas definidas por la fórmula matemática:

$$
\left(\left(\frac{x}{a}\right)^{\frac{2}{\epsilon_{2}}}+\left(\frac{y}{b}\right)^{\frac{2}{\epsilon_{2}}}\right)^{\frac{\epsilon_{2}}{\epsilon_{1}}}+\left(\frac{z}{c}\right)^{\frac{2}{\epsilon_{1}}}=1
$$

En esta fórmula, los parámetros $a, b$ y $c$ controlan las dimensiones del modelo, mientras que $\epsilon_{1}$ controla la forma del modelo en la sección transversal ortogonal al plano XY y $\epsilon_{2}$ controla la forma en la sección transversal paralela al plano XY. Las formas geométricas que se pueden generar resultan especialmente interesantes para obtener la geometría aproximada de un objeto con el propósito de manipularlo. Para obtener el modelo del objeto con esta aproximación, se debe aislar el objeto

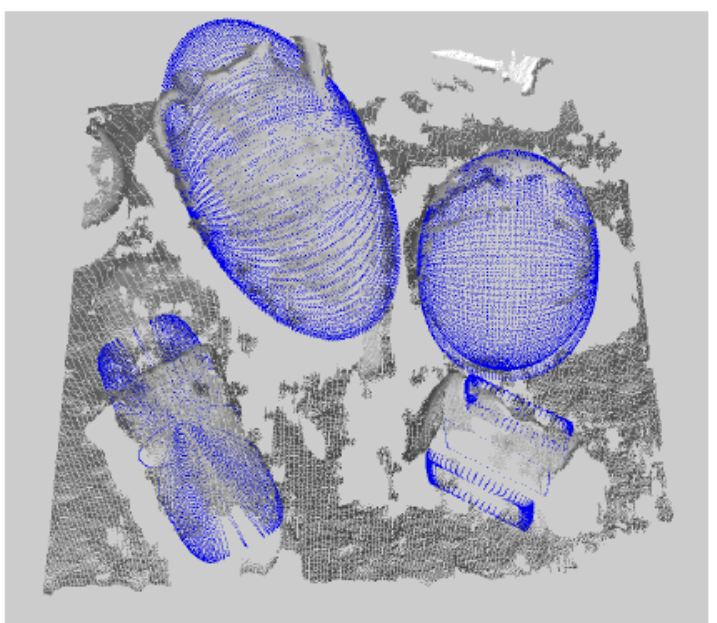

Figura 3: Reconstrucción de una escena completa utilizando diferentes parámetros de SQ para cada objeto. El modelo obtenido se muestra en azul.

del fondo y obtener una nube de puntos representativa del objeto.

Como en el caso anterior, en primer lugar se realiza una eliminación del fondo mediante RANSAC. Después, se realiza un agrupamiento por distancia euclídea para separar los diferentes objetos que aparecen en la escena. Posteriormente, proponemos la estimación de los puntos no visibles del objeto utilizando un plano de simetría (ver Figura 2). Esta asunción resulta adecuada ya que se trata de objetos hechos por el ser humano dispuestos en una superficie plana y con una visión de la escena cenital. Esto permite obtener un modelo del objeto más preciso ya que, a diferencia de RANSAC, este método no obtiene tan buenos resultados sin disponer de una entrada más precisa. Como paso previo a la obtención del modelo, se obtienen los ejes de inercia y el centroide de la nube de puntos para obtener una estimación inicial. Finamente, se obtienen los parámetros del modelo utilizando un método de minimización sobre la ecuación mostrada. Utilizando esta metodología para cada grupo de puntos obtenido se puede obtener una reconstrucción completa de la escena (ver Figura 3).

\subsection{SEGUIMIENTO DE OBJETOS}

Una vez se dispone del modelo del objeto, se realiza un seguimiento o tracking continuo del mismo utilizando los parámetros del modelo y del fondo. Utilizando la ecuación del plano correspondiente al fondo, éste se puede eliminar de manera muy eficiente. Los objetos segmentados mediante RANSAC se pueden segmentar de forma continua utilizando los parámetros dimensionales obtenidos previamente para obtener la posición y orientación nueva. En el caso de la estimación mediante 
SQ, el seguimiento es mucho más eficiente que la búsqueda del modelo por lo que esta técnica se vuelve esencial a diferencia del caso de RANSAC.

Esta metodología permite corregir errores debidos a una mala calibración y minimizar problemas como la precisión del brazo robótico o la deriva en los datos de navegación del vehículo. Además, se utiliza un filtro temporal para aumentar la robustez y descartar detecciones erróneas. Resulta significativo resaltar que este sistema es necesario para compensar el movimiento producido por las corrientes y por el movimiento inducido por el brazo al vehículo.

\section{ESPECIFICACIÓN Y EJECUCIÓN DEL AGARRE}

Una vez que los parámetros del objeto han sido obtenidos, se calcula una posición de agarre adecuada $w M g$ a través de un vector de aproximación. En el caso de un objeto cilíndrico, sea $w M o$ la posición calculada del objeto respecto al mundo, donde el eje $z$ es calculado utilizando el plano normal para dirigirlo hacia el fondo y el eje $y$ está alineado con el eje del cilindro. Usando la dirección del eje del objeto y el radio del cilindro, la transformación $o \mathrm{Mg}$, la posición de agarre con respecto al objeto, puede ser calculada heurísticamente para la obtención del mejor agarre. Esta transformación consiste en una traslación $d$ a lo largo del eje del cilindro, un ángulo de rotación $\alpha$ respecto a dicho eje y un desplazamiento radial $r$ desde el centro del cilindro $c$, tal y como se muestra en las siguientes ecuaciones:

$$
\begin{gathered}
o M g=\operatorname{Trans}_{y}(d) * \operatorname{Rot}_{x}(\alpha) * \operatorname{Trans}_{z}(r) ; \\
w M g=w M o * o M g
\end{gathered}
$$

Esta misma metodología ha sido utilizada también con esferas y cajas (ver Figura 4), considerando como eje de manipulación el eje de simetría que es paralelo al fondo. En el caso de la estimación mediante SQ se utiliza este mismo método para obtener la posición de agarre.

Con el objetivo de supervisar la manipulación, se ha desarrollado una interfaz de usuario (Figura 5). Dicha GUI, es utilizada en primer lugar para visualizar la detección de los objetos respecto a la nube de puntos, así como la posición de agarre generada automáticamente por el algoritmo. El resultado es visualizado mediante el simulador UWSim [10]. Con esta información, el usuario decide si ejecuta el agarre propuesto o lo modifica. La GUI desarrollada proporciona dos formas de modificar el agarre:

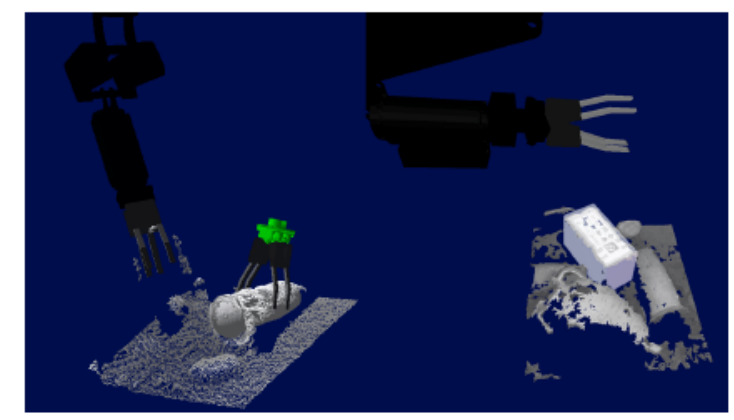

Figura 4: Detección de un cilindro (izquierda) y una caja (derecha) realizada con RANSAC. En UWSim se superpone la detección a la nube de puntos de la escena. En verde (izquierda) se muestra el resultado de la especificación del agarre.

- Respecto al objeto: el usuario puede modificar fácilmente el agarre teniendo en cuenta la forma del objeto, modificando la $r, \alpha$ y $d$ mediante intuitivas barras deslizantes.

- Marcadores interactivos: el usuario puede modificar libremente la posición 3D del objeto con marcadores interactivos (ver Figura 5). Además, también es posible ajustar la posición de manera más precisa utilizando barras deslizantes. Esta última opción proporciona más libertad al usuario pero es más difícil de utilizar para un usuario inexperto.

Esta interfaz muestra información sobre el estado de las articulaciones del brazo, los datos de navegación del vehículo y la apertura del efector final en función de las dimensiones del objeto de forma dinámica. Además, el usuario puede elegir tanto el algoritmo de detección de objetos como modificar cualquier parámetro del mismo. Si se emplea RANSAC como algoritmo de detección de objetos, desde la GUI es posible indicar el tipo de objeto a segmentar. El algoritmo SQ no requiere esta opción porque es capaz de detectar automáticamente la forma del objeto.

Cuando la posición de agarre ha sido seleccionada el usuario puede iniciar la ejecución del agarre. La

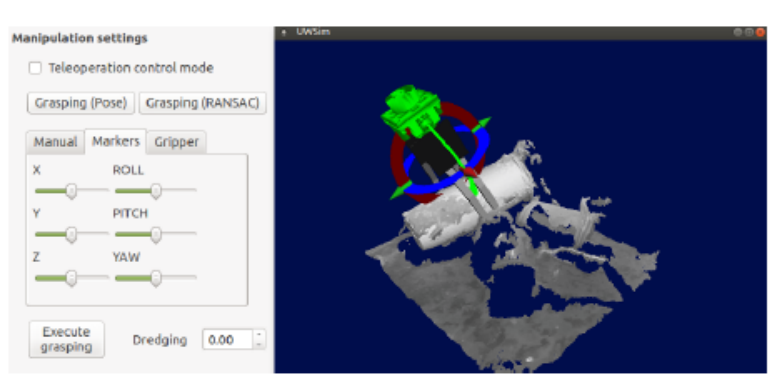

Figura 5: Especificación del agarre utilizando marcadores interactivos en $3 \mathrm{D}$. 
ejecución del robot se controla utilizando la realimentación del brazo y la visión. En este paso, la información 3D y las lecturas de corriente y encoders del brazo se utilizan para guiar el brazo y seguir el objeto. El simulador UWSim se utiliza para mostrar la posición actualizada de ambos y supervisar la operación. Si la posición y orientación del objeto cambian, la posición de agarre se corrige automáticamente. De este modo se puede reducir la incertidumbre del sistema.

\section{EXPERIMENTACIÓN Y RESULTADOS}

Los experimentos para la validación del sistema se han realizado en el tanque de agua del laboratorio IRSLab de la Universidad Jaume I. En este escenario se ha empleado el brazo ECA/CSIP Lightweight ARM5E [11] equipado con una cámara de visión estéreo (Figura 6) y acoplado a una plata-

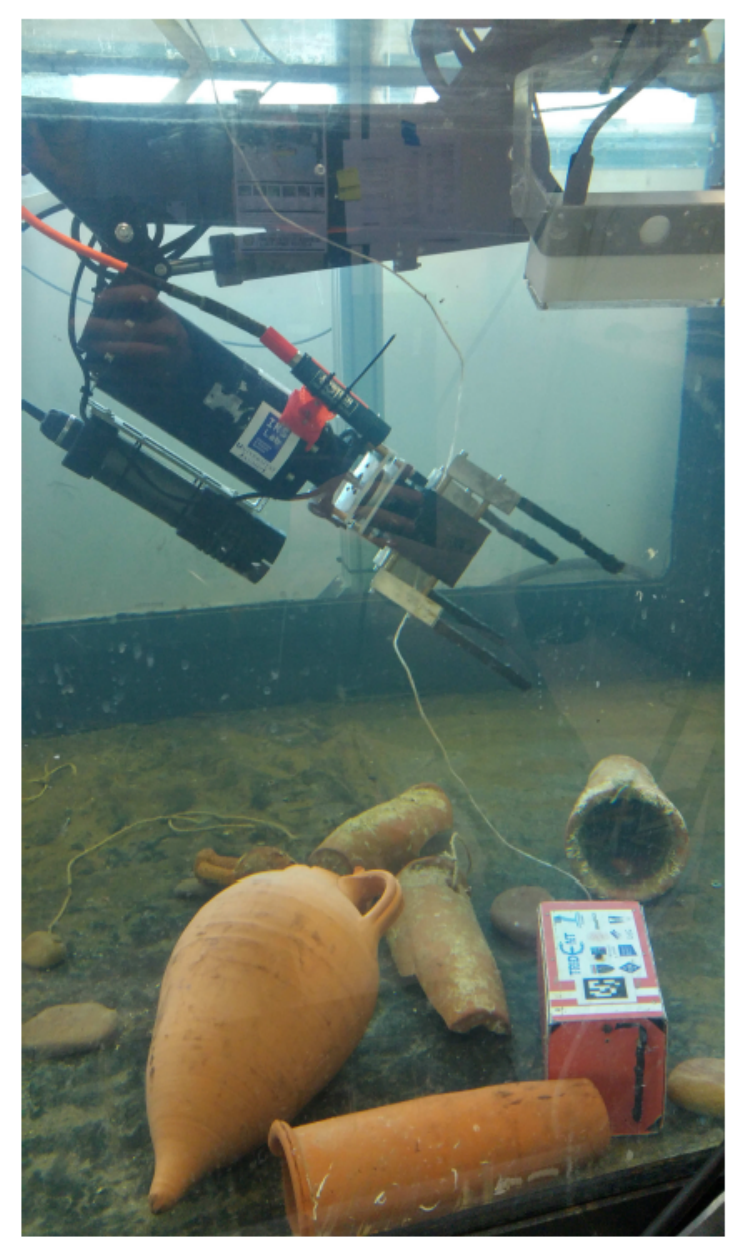

Figura 6: Condiciones de los experimentos en el tanque de agua del IRSLab. Experimentos realizados con el brazo robótico ECA/CSIP Light-weight ARM5E y una cámara estéreo en una plataforma fija.
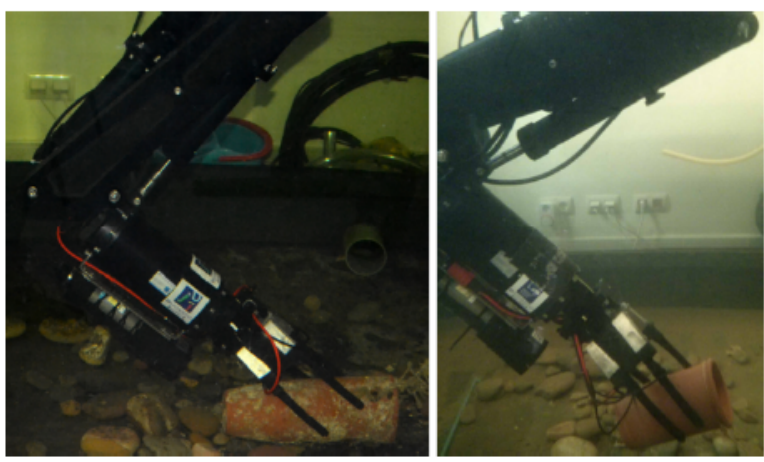

Figura 7: Experimentos de ejecución de la manipulación sobre dos objetos cilíndricos.

forma fija. Se ha llevado a cabo la estimación de vasijas y cajas de diferentes formas y dimensiones (ver Figura 3 y Figura 4). Debido a las limitaciones cinemáticas del robot, las posiciones de agarre posibles son más reducidas. Sin embargo, se han ejecutado agarres sobre diferentes vasijas satisfactoriamente, mostrándose en la Figura 7 algunos de ellos. Para llevar a cabo estos experimentos se ha monitorizado y supervisado la tarea utilizando la HRI desarrollada (ver Figura 5).

Además, el sistema de segmentación y estimación de la posición visual ha sido probado en simulación y en el mar en el contexto del proyecto MERBOTS. En ambos casos se ha empleado el vehículo Girona 500 [12] con el brazo ECA/CSIP Lightweight ARM5E [11], equipado con una cámara de visión estéreo. En estos escenarios se ha considerado el modelo de una vasija para verificar el funcionamiento del sistema de reconstrucción, segmentación, estimación del agarre y seguimiento. El simulador UWSim permite evaluar de forma realista los algoritmos de visión puesto que simula adecuadamente las cámaras estéreo, resultando ser una herramienta muy útil a la hora de validar el sistema (ver Figura 8).

Como se ha explicado en secciones anteriores, la segmentación mediante RANSAC es rápida, pudiéndose realizar a una frecuencia de hasta $10 \mathrm{~Hz}$. Por otro lado, la estimación utilizando SQ tiene un coste del orden de decenas de segundos por lo que requiere de una inicialización previa.

\section{CONCLUSIONES}

En este trabajo se ha mostrado un sistema de agarre semiautónomo, que utiliza técnicas de visión en conjunto con la inteligencia del usuario, para realizar una planificación y ejecución del agarre que permita la recuperación de objetos en el entorno submarino de forma fiable, facilitando el control y la supervisión de la misión. 


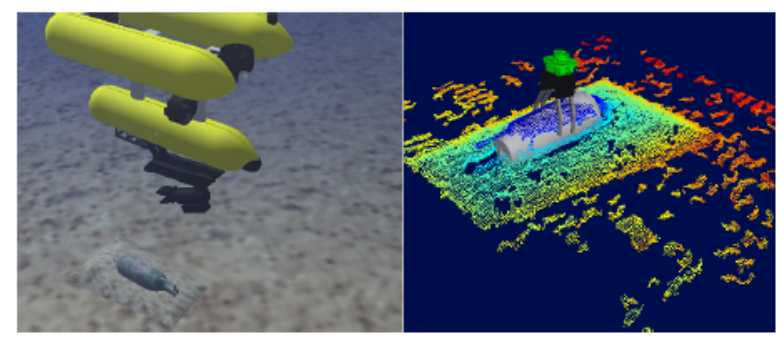

Figura 8: Escena de simulación en UWSim con una vasija (izquierda). Detección del cilindro con RANSAC y posición de agarre del objeto sobre la nube de puntos obtenida (derecha).

Se han mostrado los resultados obtenidos con objetos cilíndricos, esféricos y rectangulares en experimentos realizados en el tanque de agua de la Universitat Jaume I, en simulación y en los experimentos del proyecto MERBOTS realizados en el mar.

Entre los métodos descritos se puede destacar que los basados en RANSAC tienen una gran tolerancia al ruido, que puede ser intenso en este tipo de escenarios, y una velocidad adecuada a la frecuencia de control a alto nivel que puede requerir un robot. Sin embargo, la estimación basada en formas geométricas como son las $\mathrm{SQ}$, muestra una precisión y flexibilidad mucho mayores, dotando de una mayor fidelidad al sistema. Además, en este caso, el usuario no debe seleccionar qué tipo de forma desea estimar. Como contrapartida, es un método sensible al ruido (que pude ser filtrado) y lento (que se puede permitir en una fase de inicialización).

Como trabajo futuro se propone la utilización del resultado de la segmentación RANSAC como modelo inicial para la estimación de SQ. A través de los parámetros obtenidos mediante RANSAC es posible obtener unos parámetros iniciales para SQ que reduzcan el tiempo de minimización.

\section{Agradecimientos}

Este trabajo ha sido parcialmente financiado por el Ministerio de Economía y Competitividad (Proyecto MERBOTS DPI2014-57746-C3 y ayuda predoctoral BES-2015-073112), por la Universitat Jaume I (proyecto P11B2015-68 y ayuda predoctoral PREDOC/2013/46), y por la Generalitat Valenciana (PROMETEO/2016/066).

\section{English summary}

\section{ADVANCES IN UNDERWATER ROBOTIC MANIPULATION USING SEGMENTATION AND VISUAL TRACKING}

\section{Abstract}

This paper presents a semi-autonomous robotic system which uses vision, $3 D$ reconstruction, segmentation, object estimation, human-robot interface (HRI) and grasp planning in order to improve the underwater manipulation state-of-the-art using autonomous functionalities and supervision capabilities. Firstly, a 3D reconstruction of the object is obtained. Afterwards, RANSAC (RANdom SAmple Consensus) and superquadrics (SQ) estimation based methods are used to calculate the underlying geometry and pose of the objects to be manipulated in order to show a reconstruction of the scene to the user. Next, the grasp specification is carried out autonomously. Then, the result can be validated or tuned by the user to execute the grasp. The system has been successfully tested in different conditions both in simulation and in real environments.

Keywords: 3D reconstruction, pose estimation, superquadrics, manipulation, robotics.

\section{Referencias}

[1] P. Ridao, M. Carreras, D. Ribas, P.J. Sanz, G. Oliver, Intervention AUVs: The Next Challenge, IFAC Proceedings Volumes, Volume 47, Issue 3, 2014, Pages 12146-12159.

[2] O. Khatib, X. Yeh, G. Brantner, B. Soe, B. Kim, S. Ganguly, H. Stuart, S. Wang, M. Cutkosky, A. Edsinger, P. Mullins, M. Barham, C.R. Voolstra, K.N. Salama, M. L'Hour and V. Creuze, Ocean One: $A$ Robotic Avatar for Oceanic Discovery, in IEEE Robotics \& Automation Magazine, vol. 23, no. 4, pp. 20-29, Dec. 2016. DOI 10.1109/MRA.2016.2613281

[3] J. Bohg, A. Morales, T. Asfour and D. Kragic, Data-Driven Grasp Synthesis-A Survey, in IEEE Transactions on Robotics, vol. 30, no. 2, pp. 289-309, April 2014.

[4] J. Perez, J. Sales, A. Penalver, D. Fornas, J.J. Fernandez, J.C. Garcia, P.J. Sanz, R. Marin and M. Prats, Exploring 3-d reconstruction techniques: A benchmarking tool for 
underwater robotics, in Robotics \& Automation Magazine, IEEE, vol. 22(3), IEEE, pp. $85-95$.

[5] A. Aitor, Z. Marton, F. Tombari, W. Wohlkinger, C. Potthast, B. Zeisl, R.B. Rusu, S. Gedikli and M. Vincze, Tutorial: Point cloud library: Three-dimensional object recognition and 6 dof pose estimation, in Robotics \& Automation Magazine, IEEE, vol. 19 (3), IEEE, 2012, pp. $80-91$.

[6] J. Prankl, A. Aldoma Buchaca, A. Svejda and M. Vincze, RGB-D Object Modelling for Object Recognition and Tracking, in IEEE/RSJ International Conference on Intelligent Robots and Systems (IROS), 2015.

[7] M.A. Fischler, R.C. Bolles, Random Sample Consensus: A Paradigm for Model Fitting with Applications to Image Analysis and Automated Cartography, in Communications of the ACM Magazine, Volume 24 Issue 6, June 1981, Pages 381-395, DOI $10.1145 / 358669.358692$

[8] A.H. Barr, Superquadrics and AnglePreserving Transformations, in IEEE Computer Graphics and Applications, vol 1 (1), 1981, pp. 11-23

[9] D. Fornas, J. Sales, A. Peñalver, J. Perez, J.J. Fernández, R. Marín and P.J. Sanz, Fitting primitive shapes in point clouds: a practical approach to improve autonomous underwater grasp specification of unknown objects, in Journal of Experimental \& Theoretical Artificial Intelligence, vol 28 (1-2), 2015, pp. 369-384, DOI 10.1080/0952813X.2015.1024495

[10] M. Prats, J. Perez, J.J. Fernandez, and P.J. Sanz, An open source tool for simulation and supervision of underwater intervention missions, in 2012 IEEE/RSJ International Conference on Intelligent Robots and Systems (IROS), pp. 2577-2582, 7-12 Oct. 2012

[11] J.J. Fernández, M. Prats, P.J. Sanz, J.C. García, R. Marín, M. Robinson, D. Ribas, and P. Ridao, Grasping for the Seabed: Developing a New Underwater Robot Arm for Shallow-Water Intervention, in Robotics \& Automation Magazine, IEEE, vol. 20 (4), IEEE, 2013, pp. $121-130$.

[12] D. Ribas, N. Palomeras, P. Ridao, M. Carreras and A. Mallios, Girona 500 AUV: From Survey to Intervention, in IEEE/ASME Transactions on Mechatronics, vol. 17, no. 1, pp. 46-53, Feb. 2012.

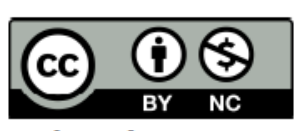

(C) 2018 by the authors. Submitted for possible open access publication under the terms and conditions of the Creative Commons Attribution CC-BY-NC 3.0 license (http://creativecommons.org/licenses/by-nc/3.0/). 